\title{
A defence of the via negativa argument for physicalism
}

\author{
Barbara Montero \& David Papineau
}

\section{The via negativa}

The key premiss in the familiar causal argument for physicalism is the Completeness of Physics (CP):

(CP) Every physical event is determined, in so far as it is determined at all, by preceding physical conditions and laws.

Given this premiss, and assuming the implausibility of systematic causal overdetermination, it follows that everything that has a physical effect must itself be physical.

However, what does 'physical' mean in this context? Defenders of CP face Hempel's Dilemma: if 'physical' means what is recognized by current physics, then $\mathrm{CP}$ is likely to be false, for historical form suggests that future research will substantially correct the ontology of current physics; on the other hand, if 'physical' is understood to refer to some future, ideal physics, then CP is irredeemably vague, since we cannot predict what future physics will bring (Hempel 1980).

David Spurrett and David Papineau (1999) have suggested that physicalists can respond by equating 'physical' with 'non-mental', thus converting the Completeness of Physics (CP) into the Completeness of the Non-Mental (CNM), and ending up with physicalism as the thesis that everything that has a non-mental effect must itself be non-mental.

Carl Gillett and Gene Witmer (2001) argue that this definitional manoeuvre does not help. In particular, they maintain that Hempel's Dilemma bites just as strongly against the 'via negativa' reading of 'phys- 
ical' as 'non-mental' as it does against ordinary physicalism. Thus they argue that 'the via negativa does not represent an advance on the original Causal Argument', and urge that 'if someone wants to use the via negativa, then she owes us an account of exactly how Hempel's Dilemma is serious when applied to CP, but not when applied to the justification of CNM' (Gillett and Witmer 2001: 303, 306). The aim of this note is to provide just such an account.

\section{Gillett and Witmer's objection}

Gillett and Witmer do not argue directly that Hempel's dilemma arises for the notion of 'non-mental' (that is, they do not argue that 'currently nonmental' makes CNM patently false, while 'ideally non-mental' makes it empty ...). On the contrary, they are perfectly happy to allow that 'mental' is an unproblematic term whose understanding raises no immediate difficulties for CNM (306). ${ }^{1}$ Rather, their worry is that Hempel's Dilemma reemerges when we consider how Spurrett and Papineau aim to support CNM. Spurrett and Papineau hold that such support comes from physiology. As Spurrett and Papineau see it,

It seems highly plausible that the non-mental is in fact complete ... To deny this is to suppose that some non-mental effects are due to irreducibly mental causes.... There is nothing incoherent or absurd in these views. But we take it that the empirical evidence, especially in the form of nineteenth- and twentieth-century physiological research, now weighs strongly against such irreducible mental causes. (1999: 26-27)

Papineau (2002) expands on this. As he says, the empirical evidence, which includes many developments in neurophysiology and the discovery of DNA,

made it difficult to go on maintaining that special forces operate inside living bodies. If there were such forces, they could be expected to display some manifestation of their presence. But detailed physiological investigation failed to uncover evidence of anything except familiar physical forces [i.e. forces that are not sui generis mental]. In this way, the argument from physiology can be viewed as clinching the case for completeness. (253-54)

To put it simply, the physiological argument for CNM is this: given that one would expect sui generis mental causes to turn up under physiological

${ }^{1}$ Still, care is needed with the notion of non-mental. In the current context, we need to understand 'non-mental' as not-sui-generis-mental, and not as not-mental-at-all, if we want to avoid making an inconsistency of the thesis that mental causes are non-mental. 
investigation, if they exist, the evidence for CNM is our failure, after extensive and prolonged physiological investigation, to come across any such mental causes.

Gillett and Witmer argue that this physiological justification of CNM is no less prey to Hempel's dilemma than the original version of CP. They argue that Spurrett and Papineau's defence of CNM commits them to the following Physiological Thesis (PT):

(PT) Bodily movement events are determined, ceteris paribus, by preceding physiological conditions and laws. (2001: 306)

Gillett and Witmer then maintain that this thesis raises just the same questions about defining 'physiological' as Hempel originally raised about 'physical'. In their words,

Either we define the 'physiological' by reference to present physiological theory or we define it by reference to some future or ideal theory. If we opt for the former, then the above claim [bodily movement events are determined, ceteris paribus, by preceding physiological conditions and laws] is likely to be false. If we opt for the latter, the claim is unacceptably vague and indeterminate. To make the latter point more dramatic, we may ask this question: Why think that the future or ideal physiological theory won't include in its ontology outright reference to the mental? In that case, the above claim cannot be used to support CNM, as the physiological conditions will include some mental conditions. (2001: 306)

\section{Response to the objection}

This would be a reasonable complaint if a defence of PT were essential to Spurrett and Papineau's project. If their argument hinged on a substantial version of PT, then they would indeed need to understand 'physiology' in such a way as to make PT both contentful and plausible, and no obvious such reading offers itself. Current physiology certainly doesn't account for all bodily movements, and the nature of ideal future physiology is uncertain.

However, there is no need to understand Spurrett and Papineau as committed to PT. Rather, their appeal to physiology can be read as simply serving to direct attention to the fact that certain kinds of empirical research have so far failed to uncover evidence of sui generis mental causes. On the basis of this failure, Spurrett and Papineau then infer, via scientific induction, that most likely there are no such sui generis mental causes.

Given this, Spurrett and Papineau can happily understand 'physiology' in their argument as referring to current physiology, rather than to any 
ideal future theory. After all, it is current physiological research that has failed to find any sui generis mental causes. But this doesn't mean that they are impaled on the first horn of Hempel's dilemma. For they have no commitment to upholding a version of PT for current physiology, that is, to claiming that current physiology has positively identified all the causes of bodily movements, which is surely false. Rather, Spurrett and Papineau's thought is simply that current physiological research has so far failed to reveal any sui generis mental causes, and from this it is reasonable to infer that there aren't any such causes. Of course, this inference is no more infallible that any other inductive inference, but Spurrett and Papineau are not claiming that it is. (Cf. the passage quoted above: '... we take it that the empirical evidence ... weighs strongly against such irreducible mental causes.')

Perhaps there is room to query whether lack of evidence for sui generis mental causes should count as positive evidence against the existence of such causes. To be sure, there are cases where lack of evidence for X does seem to provide evidence that $\mathrm{X}$ does not exist. For example, we reject the idea that ghosts exist in part because we have never found anything that could be identified as a ghost. On the other hand, there also seem to be cases where failure to find evidence for $\mathrm{X}$ does not count as sufficient evidence against X. For example, the fact that I've never seen bats in my cellar is not good evidence that there are none, if I've only been in my cellar during the day.

Maybe the physiological evidence against sui generis mental causes suffers from a similar flaw. Perhaps the only reason physiologists haven't uncovered any evidence is that they have been looking in the wrong place. To switch analogies, maybe physiologists are like the fellow searching for his keys under the street light even though he lost them in the dark alley - that is, perhaps physiological researchers are limited by their exclusive commitment to familiar but ineffective means of investigation.

However, Spurrett and Papineau have no real need to defend the adequacy of physiology's exploratory strategies. If the thought is that nonphysiological modes of investigation might uncover sui generis mental causes, they can simply respond that no such modes of investigation have succeeded in doing so thus far, and that this again provides inductive evidence against mental causes. The essential point is not so much that physiology has failed to find mental causes, but that no type of empirical investigation has. (Doesn't first-person experience reveal mental causes? Maybe so. But it is another thing to show that it reveals sui generis mental causes.)

In any case, we need not pursue the adequacy of the inductive evidence against sui generis mental forces any further. The point of this note was not to vindicate this inductive argument, which would be a substantial 
empirical task, but merely to establish the far more modest point that Hempel's Dilemma does not undermine the via negativa and the associated appeal to physiological evidence. ${ }^{2}$

City University of New York The Graduate Center and The College of Staten Island 365 Fifth Avenue New York, NY 10016-4309, USA bmontero@gc.cuny.edu

King's College London, Strand London WC2R $2 L S$, UK david.papineau@kcl.ac.uk

\section{References}

Gillett, C. and G. Witmer. 2001. A physical need: physicalism and the via negativa. Analysis 61: 302-308.

Hempel, C. 1980. Comments on Goodman's Ways of Worldmaking. Synthese 45: 19399.

Papineau, D. 2002. Thinking about Consciousness. Oxford: Oxford University Press.

Spurrett, D. and D. Papineau. 1999. A note on the completeness of 'physics'. Analysis 59: 25-29.

2 The first author's work on this paper was supported by an NEH grant. 